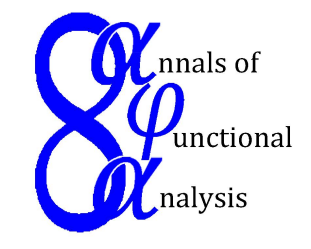

Ann. Funct. Anal. 6 (2015), no. 3, 53-59

http://doi.org/10.15352/afa/06-3-5

ISSN: 2008-8752 (electronic)

http://projecteuclid.org/afa

\title{
PERTURBATIONS OF THE BALL ALGEBRA
}

\author{
KRZYSZTOF JAROSZ
}

Communicated by M. S. Moslehian

\begin{abstract}
We prove that any Banach algebra that is geometrically close to a Ball Algebra must automatically consist of analytic functions and must have a very similar algebraic structure to the original algebra.
\end{abstract}

\section{INTRODUCTION}

Let $A$ be a Banach algebra and let $B$ be a small deformation of $A$. What properties $A$ and $B$ have to share? Do they have to be automatically isomorphic? These and related questions have been investigated for many years by a number of authors, see for example $[2,3,7,8]$. The answers obviously depend on the exact definition of a "small deformation". The two simplest and most fundamental definitions of a deformation refer to metric or to algebraic structures of the algebras:

Definition 1.1. A Banach algebra $B$ is a metric $\delta$-deformation of a Banach algebra $A$ if there is a linear (but not necessarily multiplicative) isomorphism $T: A \rightarrow B$ such that $\|T\|\left\|T^{-1}\right\| \leq 1+\delta$.

Definition 1.2. For a Banach algebra $(A, \cdot)$ a new multiplication $\times$ defined on the same Banach space $A$ is an algebraic $\delta$-deformation of $(A, \cdot)$ if $\|\times-\cdot\| \leq \delta$; that is, if

$$
\|a \cdot b-a \times b\| \leq \delta\|a\|\|b\|, \quad \text { for } a, b \in A .
$$

While the two definitions lead to different theories for general Banach algebras, they are equivalent in a natural way for all uniform algebras, that is for closed

Date: Received: Dec. 8, 2014; Accepted: Dec. 15, 2014.

2010 Mathematics Subject Classification. Primary 46J15; Secondary 32G15, 46 E15.

Key words and phrases. Banach algebra, uniform algebra, ball algebra, perturbation, deformation. 
subalgebras of $C(X)$, the algebra of continuous complex valued functions defined on a compact set $X$ (see Theorem 3.7 below).

There are several important links between the deformation theory and other areas. For example it provides a natural definition of a deformation of an analytic manifold, or a domain $\Omega$ in $\mathbb{C}^{n}$. We may define the distance between two domains $\Omega$ and $\Omega^{\prime}$ by

$$
d\left(\Omega, \Omega^{\prime}\right)=\inf \left\{\|T\|\left\|T^{-1}\right\|: \quad T: A(\Omega) \rightarrow A\left(\Omega^{\prime}\right)\right\},
$$

where $A(\Omega)$ is a Banach space of analytic functions on $\Omega$. It is an important and deep result due to $\mathrm{R}$. Rochberg [7] that for one dimensional Riemann surfaces the distance defined above is locally equivalent to the Teichmüller distance involving quasiconformal homeomorphisms. Still, almost nothing is known about domains in $\mathbb{C}^{n}$ for $n>1$, we only had a partial result about the Polydisc Algebra [3]. In this note we prove that a small deformation of a Ball Algebra

$$
A\left(B^{n}\right)=\left\{f \in C\left(\overline{B^{n}}\right): f \text { is analytic on } \operatorname{int} B^{n}\right\},
$$

where $B^{n}$ is the unit ball in $\mathbb{C}^{n}$, must automatically share a lot of the structure with the original algebra.

\section{The Main Result}

Theorem 2.1. Let $A\left(B^{n}\right)$ be a Ball Algebra and let $B$ be its $\varepsilon$ metric deformation with $\varepsilon<\varepsilon_{0}$. Then $B$ is a uniform algebra whose maximal ideal space $\mathfrak{M}(B)$ is homeomorphic with $\overline{B^{n}}$. Furthermore int $B^{n}$ can be given a structure $\sigma$ of an $n$ dimensional analytic manifold such that all functions from $B$ are analytic on $(B, \sigma) ; \varepsilon_{0}>0$ is an absolute constant.

\section{Preliminary Results and Notation}

The proof of the theorem is given in the next section. In this section we state several preliminary results which will be used in that proof. All these results are well known or easily follow from known results. First we need to establish some notation.

Definition 3.1. For a positive number $r$ we denote by $\Delta^{n}(\mathbf{0}, r)$ the polycylinder with radius $r$ defined by

$$
\Delta^{n}(\mathbf{0}, r) \stackrel{d f}{=}\left\{\left(z_{1}, \cdots, z_{n}\right) \in \mathbb{C}^{n}:\left|z_{k}\right|<r \text { for } k=1,2, \cdots, n\right\} .
$$

Definition 3.2. For a commutative Banach algebra $A$ we denote by $\mathfrak{M}(A)$ the set of all maximal ideals on $A$; we often identify such an ideal with the corresponding linear and multiplicative functional and we assume that $\mathfrak{M}(A)$ is equipped with the weak $*$ topology.

Definition 3.3. A linear functional $F$ on a Banach algebra $A$ is called $\delta$-multiplicative if

$$
|F(a \cdot b)-F(a) F(b)| \leq \delta\|a\|\|b\| \text { for all } a, b \in A \text {. }
$$


Definition 3.4. For $\boldsymbol{z}, \boldsymbol{w} \in \mathbb{C}^{n}$ we put

$$
\|\boldsymbol{w}\|_{\infty}=\max _{k}\left|w_{k}\right|,\langle\boldsymbol{z}, \boldsymbol{w}\rangle=\sum_{k=1}^{n} z_{k} \bar{w}_{k}, \text { and } P_{\boldsymbol{z}}=\frac{\langle\boldsymbol{z}, \boldsymbol{w}\rangle}{\|\boldsymbol{w}\|^{2}} \boldsymbol{w} .
$$

Theorem 3.5. Let $A$ be a unital, complex uniform algebra and let $F$ be a linear and multiplicative functional on $A$. Assume that there is a constant $C$ and norm one elements $f_{1}, \cdots, f_{n}$ in $A$ such that for any $f \in \operatorname{ker} F$ there are $h_{1}, \cdots, h_{n}$ in $A$ such that $f=\sum_{k=1}^{n} h_{k} f_{k}$ and $\left\|h_{k}\right\| \leq C$ for $k=1,2, \cdots, n$. Then there is a positive number $r$ which depends only on $C$ and $n$, and an analytic variety $V$ contained in a polycylinder $\Delta^{n}(\mathbf{0}, r)$ such that $\tau(G) \stackrel{d f}{=}\left(G\left(f_{1}\right), \cdots, G\left(f_{n}\right)\right)$ is a homeomorphism from

$$
U \stackrel{d f}{=}\left\{G \in \mathfrak{M}(A):\left|G\left(f_{k}\right)\right|<r \text { for } k=1,2, \cdots, n\right\}
$$

onto $V$. Furthermore $f \circ \tau^{-1}$ extends analytically to $\Delta^{n}(\mathbf{0}, r)$ for all $f \in A$.

Proof. The above result is a version of the classical Gleason Embedding Theorem; while the standard version of this theorem does not include such specific description of $\tau, r$, and $U$ this is how these objects are defined in the proof ([5] pages 154-155).

The next two theorems are taken from [4] and [2] respectively.

Theorem 3.6. Let $F$ be a $\delta$-multiplicative functional on a Ball Algebra $A\left(B^{n}\right)$ then there is $G \in \mathfrak{M}\left(A\left(B^{n}\right)\right)$ such that $\|F-G\| \leq \varepsilon$, where $\varepsilon \rightarrow 0$ as $\delta \rightarrow 0$.

Theorem 3.7. Let $T: A \rightarrow B$ be a linear bijection from a uniform algebra $A$ onto a Banach algebra $B$ such that $\|T\|\left\|T^{-1}\right\| \leq 1+\delta$ where $\delta$ is smaller that an absolute constant $\delta_{0}>0$. Then

- $T \mathbf{1}$ is an invertiable element of $B$ and $\widetilde{T} \stackrel{d f}{=}(T(\mathbf{1}))^{-1} T$ preservs the units of the algebras and is such that $\|\widetilde{T}\| \leq 1+3 \delta$ and $\left\|\widetilde{T}^{-1}\right\| \leq 1+3 \delta$,

- if a new multiplication $\times$ on $A$ is defined by $a \times b=\widetilde{T}^{-1}(\widetilde{T} a \cdot \widetilde{T} b)$ then $\times$ is a $\delta^{\prime}$ algebraic deformation of $A$,

- $B$ is a uniform algebra and there is a homeomorphism $\varphi$ from the Choquet boundary $C h B$ of $B$ onto the Choquet boundary ChA of $A$ such that

$$
|\widetilde{T} f(\boldsymbol{x})-f(\varphi(\boldsymbol{x}))| \leq \delta^{\prime}\|f\| \text { for any } \boldsymbol{x} \in C h B \text { and } f \in A,
$$

where the constants $\delta, \delta^{\prime}$ tend to 0 simultaneously.

One of the minor consequences of the above Theorem is that we may assume without loss of generality when discussing small deformations that $T \mathbf{1}=\mathbf{1}$.

The following Proposition can be found in [6], page 391.

Proposition 3.8. Let $\boldsymbol{w}=\left(w_{1}, \cdots, w_{n}\right) \in \operatorname{int} B^{n}$ and define a function $\Psi_{\boldsymbol{w}}$ : $\bar{B}^{n} \rightarrow \mathbb{C}^{n}$ by

$$
\Psi_{\boldsymbol{w}}(\boldsymbol{z})=\frac{\boldsymbol{w}-P_{\boldsymbol{z}}-\sqrt{1-\|\boldsymbol{w}\|^{2}}\left(\boldsymbol{z}-P_{\boldsymbol{z}}\right)}{1-\langle\boldsymbol{z}, \boldsymbol{w}\rangle},
$$

Then $\Psi_{\boldsymbol{w}}$ is an analytic automorphism of $B^{n}$ such that $\Psi_{\boldsymbol{w}}(\boldsymbol{w})=\mathbf{0}$. 
Theorem 3.9. Let $r>0$ and let $\psi: \Delta^{n}(\mathbf{0}, 2 r) \rightarrow \Delta^{n}(\mathbf{0}, 3 r)$ be a continuous function such that

$$
\|\psi(p)-p\|_{\infty} \leq r \text { for all } p
$$

then the range of $\psi$ contains $\Delta^{n}(\mathbf{0}, r)$.

Proof. The result is an easy consequence of the Brouwer Fix Point Theorem. By (3.1) for all $p \in \Delta^{n}(\mathbf{0}, 2 r) \backslash \Delta^{n}(\mathbf{0}, r)$ we have $\psi(p) \neq-p$, hence the map

$p \rightarrow \frac{\psi(p)}{\|\psi(p)\|_{\infty}}$ restricted to the boundary of $\Delta^{n}\left(\mathbf{0}, r^{\prime}\right)$ for $r<r^{\prime}<2 r$ is homotopic with the identity map, hence it is surjective.

\section{Proof of the Main Theorem}

Assume that a Banach algebra $B$ is a small deformation of $A\left(B^{n}\right)$. By Theorem 3.7 there is a linear bijection $T: A\left(B^{n}\right) \rightarrow B$ such that $\|T\| \leq 1+\varepsilon,\left\|T^{-1}\right\| \leq 1+\varepsilon$, and $T \mathbf{1}=\mathbf{1}$. By the same Theorem $B$ is a uniform subalgebra of $C(X)$, where $X=\mathfrak{M}(B)$, and $X$ contains a copy the unit sphere $\partial B^{n}$ which is equal to the Choquet boundary of $B$, furthemore

$$
|T f(\boldsymbol{z})-f(\boldsymbol{z})| \leq \varepsilon\|f\| \text { for } \boldsymbol{z} \in \partial B^{n} \text { and } f \in A\left(B^{n}\right),
$$

where we use the same symbol $\boldsymbol{z}$ for a point in $\partial B^{n}$ and for the corresponding point in the homeomorphic copy of $\partial B^{n} \subset X$. Furthermore, adjusting the $\varepsilon$ if necessary, by Theorem 3.7 we also have

$$
\left\|T\left(f_{1} \cdot f_{2}\right)-T\left(f_{1}\right) T\left(f_{2}\right)\right\| \leq \varepsilon\left\|f_{1}\right\|\left\|f_{2}\right\| \text { for } f_{1}, f_{2} \in A\left(B^{n}\right) .
$$

The next Lemma shows that (4.1) can be extended to include $\boldsymbol{z} \in$ int $B^{n}$.

Lemma 4.1. There is a function $\varphi:$ int $B^{n} \rightarrow X$ such that

$$
|T f(\varphi(\boldsymbol{z}))-f(\boldsymbol{z})| \leq c \varepsilon\|f\| \text { for } \boldsymbol{z} \in \operatorname{int} B^{n} \text { and } f \in A\left(B^{n}\right),
$$

where $c$ is a constant depending only on $n$.

Proof of the Lemma. We first assume that $z=\mathbf{0}=(0, \cdots, 0)$; we claim that

$$
\varphi(\mathbf{0}) \stackrel{d f}{=}\left\{\sum_{k=1}^{n} T\left(Z_{k}\right) \cdot g_{k}: g_{k} \in B\right\}
$$

is a codimension one ideal in $B$; here $Z_{k}$ is a function in $A\left(B^{n}\right)$ defined by $Z_{k}\left(z_{1}, z_{2}, \cdots, z_{n}\right)=z_{k}$.

By [1] (see also [9] pp. 151-153), a linear map $\Phi$ from a product of $n$ copies of $A\left(B^{n}\right)$ into

$$
A_{0}\left(B_{n}\right) \stackrel{d f}{=}\left\{f \in A\left(B_{n}\right): f(\mathbf{0})=0\right\},
$$

defined by

$$
\Phi\left(f_{1}, \cdots, f_{n}\right)=\sum_{k=1}^{n} Z_{k} f_{k},
$$

is surjective. Hence, by the Open Mapping Theorem there is a constant $C$ such that for any $f \in A_{0}\left(B^{n}\right)$ there are $\Phi_{k} f \stackrel{d f}{=} f_{k} \in A\left(B_{n}\right)$ with $\left\|f_{k}\right\| \leq C\|f\|$ and such that $\Phi\left(f_{1}, \cdots, f_{n}\right)=f$. 
We define $S: A\left(B^{n}\right) \rightarrow B \subset C(X)$ by

$$
S_{\mathbf{0}}(f)=f(\mathbf{0})+\sum_{k=1}^{n} T\left(Z_{k}\right) T\left(\Phi_{k}(f-f(\mathbf{0}))\right) .
$$

For any function $f$ in $A\left(B^{n}\right)$ we have

$$
f=f(\mathbf{0})+\sum_{k=1}^{n} Z_{k} \Phi_{k}(f-f(\mathbf{0}))
$$

hence by (4.2) and since $T \mathbf{1}=\mathbf{1}$ we get

$$
\begin{aligned}
\left\|T f-S_{\mathbf{0}} f\right\| & =\left\|T\left(\sum_{k=1}^{n} Z_{k} \Phi_{k}(f-f(\mathbf{0}))\right)-\left(\sum_{k=1}^{n} T\left(Z_{k}\right) T\left(\Phi_{k}(f-f(\mathbf{0}))\right)\right)\right\| \\
& \leq \sum_{k=1}^{n}\left\|T\left(Z_{k} \Phi_{k}(f-f(\mathbf{0}))\right)-T\left(Z_{k}\right) T\left(\Phi_{k}(f-f(\mathbf{0}))\right)\right\| \\
& \leq 2 n C \varepsilon\|f\| .
\end{aligned}
$$

Hence

$$
\left\|S_{\mathbf{0}}(f)\right\| \geq\|T f\|-2 n C \varepsilon\|f\| \geq\left(\frac{1}{1+\varepsilon}-2 n C \varepsilon\right)\|f\| .
$$

If $2 n C \varepsilon$ is sufficiently small it follows that $S_{\mathbf{0}}$ is an isomorphism from $A\left(B^{n}\right)$ onto $B$ so it maps a codimension one subspace $A_{0}\left(B_{n}\right)$ onto codimension one ideal $\varphi(\mathbf{0})$. We also get

$$
\left\|I d-S_{\mathbf{0}}^{-1} \circ T\right\| \leq\left\|T-S_{\mathbf{0}}\right\|\left\|S_{\mathbf{0}}^{-1}\right\| \leq c \varepsilon
$$

where $c$ is a constant depending on $n$. Since

$$
S_{\mathbf{0}}(f)(\varphi(\mathbf{0}))=f(\mathbf{0}) \text { or equivalently } g(\varphi(\mathbf{0}))=S_{\mathbf{0}}^{-1}(g)(\mathbf{0})
$$

we also get that

$$
|T(f)(\varphi(\mathbf{0}))-f(\mathbf{0})| \leq\left|S_{\mathbf{0}}^{-1}(T f)(\mathbf{0})-f(\mathbf{0})\right| \leq c \varepsilon\|f\| .
$$

Now fix $\boldsymbol{w}=\left(w_{1}, \cdots, w_{n}\right) \in \operatorname{int} B^{n}$ and let $\Psi_{\boldsymbol{w}}$ be the automorphism defined in Proposition 3.8. Put

$$
\varphi(\boldsymbol{w}) \stackrel{d f}{=}\left\{\sum_{k=1}^{n} T\left(Z_{k} \circ \Psi_{\boldsymbol{w}}\right) \cdot g_{k}: g_{k} \in B\right\}
$$

and let $S_{\boldsymbol{w}}: A\left(B^{n}\right) \rightarrow B$ be defined by

$$
S_{\boldsymbol{w}}(f)=f(\boldsymbol{w})+\sum_{k=1}^{n} T\left(Z_{k} \circ \Psi_{\boldsymbol{w}}\right) T\left(\Phi_{k}(f-f(\boldsymbol{w}))\right) .
$$

Since we are simply replacing $T$ with a map $f \rightarrow T\left(f \circ \Psi_{\boldsymbol{w}}\right)$, exactly as before we check that

$$
\left\|T f-S_{\boldsymbol{w}} f\right\| \leq 2 n C \varepsilon\|f\|, \quad\left\|I d-S_{\boldsymbol{w}}^{-1} \circ T\right\| \leq c \varepsilon,
$$

that $\varphi(\boldsymbol{w})$ is an ideal of codimension one, that

$$
g(\varphi(\boldsymbol{w}))=S_{\boldsymbol{w}}^{-1}(g)(\boldsymbol{w})
$$


and

$$
|T(f)(\varphi(\boldsymbol{w}))-f(\boldsymbol{w})| \leq c \varepsilon\|f\|
$$

The above Lemma defines $\varphi$ on the interior of the unit ball, we extend $\varphi$ to the boundary of the ball by setting $\varphi(\boldsymbol{z})=\boldsymbol{z}$ for $z \in \partial B^{n}$.

Lemma 4.2. $\varphi: \bar{B}^{n} \rightarrow \mathfrak{M}(B)$ is continuous.

Proof of the Lemma. By (4.3) and since $S_{\boldsymbol{w}}$ depends continuously on $\boldsymbol{w}$ it follows that $\varphi$ is continuous on int $B^{n}$. Assume that $\boldsymbol{w}_{n}$ is a sequence of points in int $B^{n}$ convergent to a point $\boldsymbol{w}_{0}$ on the boundary of the ball. Assume further that $\varphi\left(\boldsymbol{w}_{n}\right)$ is not convergent to $\boldsymbol{w}_{0}$. Since $X$ is a compact metric space the sequence $\varphi\left(\boldsymbol{w}_{n}\right)$ has a subsequence convergent to $\boldsymbol{w}^{\prime} \neq \boldsymbol{w}_{0}$. To simplify the notation we assume that $\varphi\left(\boldsymbol{w}_{n}\right) \rightarrow \boldsymbol{w}^{\prime}$. By the previous Lemma we have

$$
\left|T(f)\left(w_{n}\right)-f\left(w_{n}\right)\right| \leq c \varepsilon\|f\|
$$

so by $(4.1)$

$$
\left|T(f)\left(w^{\prime}\right)-f\left(w_{0}\right)\right| \leq c \varepsilon\|f\| \text { and }\left|T(f)\left(w^{\prime}\right)-f\left(w^{\prime}\right)\right| \leq \varepsilon\|f\| .
$$

This means that the norm of the difference between functionals on $B$ "evaluation at $w^{\prime \prime}$ and "evaluation at $w_{0}$ " is not greater than $(1+c) \varepsilon$. On the other hand the norm distance between a functional from the Choquet boundary of any uniform algebra and any other linear-multiplicative functional is equal to 2 ([5]). We get a contradiction (assuming $\varepsilon$ is sufficiently small) proving continuity of $\varphi$.

Lemma 4.3. The function $\varphi$ is a surjection from $\bar{B}^{n}$ onto $X=\mathfrak{M}(B)$.

Proof of the Lemma. We prove the Lemma in two steps. We first show that for any $x$ in $X$ there is a point $\boldsymbol{z}$ in $\bar{B}^{n}$ such that $\varphi(\boldsymbol{z})$ is close to $x$. Then we show that for any $\boldsymbol{z}, \varphi\left(\bar{B}^{n}\right)$ contains all points in $X$ which are close to $\varphi(\boldsymbol{z})$.

Let $x$ be a point in $X$ and define

$$
F: A\left(B^{n}\right) \rightarrow \mathbb{C} \text { by } F(f)=T(f)(x) .
$$

By 4.2

$$
\left|F\left(f_{1} \cdot f_{2}\right)-F\left(f_{1}\right) F\left(f_{2}\right)\right| \leq \varepsilon\left\|f_{1}\right\|\left\|f_{2}\right\| \text { for } f_{1}, f_{2} \in A\left(B^{n}\right) .
$$

hence by Theorem 3.6 there is $\boldsymbol{z} \in \mathfrak{M}\left(A\left(B^{n}\right)\right)=\bar{B}^{n}$ such that

$$
|F(f)-f(\boldsymbol{z})| \leq k \varepsilon\|f\| .
$$

By $(4.4) \varphi(\boldsymbol{z})$ is close to $x$ :

$$
|g(x)-g(\varphi(\boldsymbol{z}))| \leq c k \varepsilon \text { for } g \in B
$$

This concludes the first part of the proof.

For the second part of the proof assume that $c$ and $Z_{1}, \cdots, Z_{n}$ are as in the proof of the previous Lemma, put

$$
U \stackrel{d f}{=}\left\{G \in \mathfrak{M}(B):\left|G\left(T Z_{k}\right)\right|<3 c \varepsilon \text { for } k=1,2, \cdots, n\right\},
$$


and define $\tau: U \rightarrow \Delta^{n}(\mathbf{0}, 3 c \varepsilon)$ by

$$
\tau(G) \stackrel{d f}{=}\left(G\left(T Z_{1}\right), \cdots G\left(T Z_{n}\right)\right) .
$$

Notice that $T Z_{k}(\varphi(\mathbf{0}))=0$ so $\varphi(\mathbf{0}) \in U$ and $\tau \circ \varphi(\mathbf{0})=\mathbf{0}$. Furthermore by (4.4) $\varphi(\boldsymbol{w}) \in U$ for any $\boldsymbol{w} \in \Delta^{n}(\mathbf{0}, c \varepsilon)$; for any such $\boldsymbol{w}$ we also have

$$
\begin{aligned}
& \|\tau \circ \varphi(\boldsymbol{w})-f(\boldsymbol{w})\|_{\infty} \\
= & \left\|\left(T Z_{1}(\varphi(\boldsymbol{w}))-Z_{1}(\boldsymbol{w}), \cdots, T Z_{n}(\varphi(\boldsymbol{w}))-Z_{n}(\boldsymbol{w})\right)\right\|_{\infty} \leq c \varepsilon .
\end{aligned}
$$

Hence $\tau \circ \varphi$ is a continuous map from $\Delta^{n}(\mathbf{0}, 2 c \varepsilon)$ into $\Delta^{n}(\mathbf{0}, 3 c \varepsilon)$ and by Theorem $(3.9)$

$$
\Delta^{n}(\mathbf{0}, c \varepsilon) \subset \tau \circ \varphi\left(\Delta^{n}(\mathbf{0}, 2 c \varepsilon)\right) \subset \Delta^{n}(\mathbf{0}, 3 c \varepsilon) .
$$

Assume $G \in \mathfrak{M}(B)$ is such that $\|G-\varphi(\mathbf{0})\|<c \varepsilon$ and that $G$ is not in the range of $\varphi$. Then $\tau(G) \in \Delta^{n}(\mathbf{0}, c \varepsilon)$ so there is $p \in \Delta^{n}(\mathbf{0}, 2 c \varepsilon)$ such that $\tau(\varphi(p))=\tau(G)$, since $G$ is not in the range of $\varphi$ and $\varphi(p)$ is in that range this shows that $\tau$ is not injective contrary to Theorem 3.5.

The above arguments show that any $G \in \mathfrak{M}(B)$ that is close to $\varphi(\mathbf{0})$ must be in the range of $\varphi$. For $\boldsymbol{w} \in \operatorname{int} B^{n}$ put $T_{\boldsymbol{w}}(f) \stackrel{d f}{=} T\left(f \circ \Psi_{\boldsymbol{w}}\right)$ where $\Psi_{\boldsymbol{w}}$ is the automorphism of $B^{n}$ defined in the proof of the previous Lemma. If we repeat the above arguments with $T$ replaced by $T_{\boldsymbol{w}}$ we get that any $G \in \mathfrak{M}(B)$ that is close to $\varphi(\boldsymbol{w})$ must be in the range of $\varphi$.

\section{REFERENCES}

1. J. Bruna and J.M. Ortega, Closed finitely generated ideals in algebras of holomorphic functions and smooth to the boundary in strictly psedoconvex domains, Math. Ann. 268 (1984), $137-157$.

2. K. Jarosz, Perturbations of Banach Algebras, Springer-Verlag, Lecture Notes in Math. 1120, 1985.

3. K. Jarosz, Small perturbations of algebras of analytic functions on polydiscs, In Function Spaces, Marcel Dekker, 1991, 223-240.

4. K. Jarosz, Almost multiplicative functionals, Studia Math. 124 (1997), 37-58.

5. T.W. Gamelin, Uniform Algebras, $2^{\text {nd }}$ Ed., Chelsea Pub. Comp., New York, 1984.

6. S.G. Krantz, Function Theory of Several Complex Variables, John Willey \& Sons, 1982.

7. R. Rochberg, Deformation of uniform algebras on Riemann surfaces, Pacific J. Math. 121 (1985), 135-181.

8. S.J. Sidney, Are all uniform algebras AMNM?, Bull. London Math. Soc. 29 (1997), 327-330.

9. E.L. Stout, The Theory of Uniform Algebras, Bogden \& Quigley, California, 1971.

Department of Mathematics and Statistics, Southern Illinois University EdWARDSVILlE, 62026 ILLINOIS, USA.

E-mail address: kjarosz@siue.edu 